\title{
ANÁLISE COMPARATIVA DO CRESCIMENTO DO CAMORIM, Centropomus undecimalis (BLOCH, 1792), ALIMENTADO COM DIETAS VIVA E INERTE EM VIVEIRO ESTUARINO
}

\author{
Romero Francisco de Barros ALMEIDA \\ PIBIC/CNPq/UFRPE \\ Antonio Lisboa Nogueira da SILVA \\ Departamento de Pesca da UFRPE - CNPq \\ José Arlindo PEREIRA \\ Departamento de Oceanografia da UFPE - CNPq
}

\begin{abstract}
RESUMO
Com o objetivo de verificar o grau de aceitabilidade de dieta natural inerte pelo camorim Centropomus undecimalis foi realizado um experimento na Base de Piscicultura Estuarina da Universidade Federal de Pernambuco, localizada na Ilha de Itamaracá (PE), no período de 05 de outubro de 1995 a 03 de maio de 1996. Para isto, um viveiro de 450 $\mathrm{m}^{2}$ foi dividido por cercados de tela plástica em quatro compartimentos de $60 \mathrm{~m}^{2}$. Em cada um deles foram estocados 48 camorins juvenis capturados no ambiente estuarino, com comprimento total variando de 12 a $19 \mathrm{~cm}$ e peso total médio de $30 \mathrm{~g}$. Na alimentação foram utilizados organismos provenientes da fauna do próprio estuário, apresentando dois tratamentos: A- dieta viva e, B-dieta inerte, constituída desses mesmos organismo triturados, ambos fornecidos até a saciação. No decorrer do experimento foram realizadas amostragens periódicas dos comprimentos e pesos totais dos peixes. A água do viveiro foi renovada quinzenalmente em pelo menos $80 \%$, por ocasião da preamares. A salinidade variou de 15,6 a 39\%o; a temperatura oscilou entre 28 a $35^{\circ} \mathrm{C}$ e a taxa de sobrevivência nas quatro parcelas experimentais variou de 73 a 100\%. A taxa de crescimento específico variou de 0,58 a 0,94. A taxa de conversão alimentar aparente média no tratamento $\mathbf{A}$ foi de 10,1:1 e de 8,95:1 no tratamento $\mathbf{B}$. O peso médio final do tratamento com dieta inerte $(184,6 \mathrm{~g})$ superou o da dieta viva $(114,4 \mathrm{~g})$ em $65 \%$, o que sugere a boa adequação dessa espécie a esse tratamento, no entanto, outros estudos testando manejos com vista a aumentar a taxa de crescimento e diminuir a de conversão alimentar, são recomendados.

Palavras chave: camorim, Centropomidae, alimentação, cultivo e crescimento.
\end{abstract}

\section{ABSTRACT \\ Comparative Analyses of the (Centropomus undecimalis, Bloch, 1792) Growth Fed with} Live and Non-live Diets in Estuarine Pond

The experience was conduced in an earthen pond of $450 \mathrm{~m}^{2}$, which was divided in four compartments, each one of $60 \mathrm{~m}^{2}$, separated by $5 \mathrm{~mm}$ black plastic fencing, 
supported by wood pickets buried in the ground. In each compartment, called $A_{I}$ and $\mathrm{A}_{\mathrm{II}}$, the live food treatment (A) was applied and in the $\mathrm{B}_{\mathrm{I}}$ and $\mathrm{B}_{\mathrm{II}}$ compartments the nonlive food treatment (B) was used. Wild snooks were stocked, with total length varying from 12 to $19 \mathrm{~cm}$ and $0.8 \mathrm{fish} / \mathrm{m}^{2}$ of density, totalizing 192 individuals. During the experiment, total length and weight were measured of 10 individuals at least in each experimental unit. The water salinity varied between 15.6 and $39 \%$, and the temperature from $28^{\circ} \mathrm{C}$ to $35^{\circ} \mathrm{C}$; the survival rate was between 72 and $100 \%$. On the treatment $\mathrm{A}$, the specific growth rate (SGR) varied from 0.58 and 0.64 while on treatment $\mathrm{B}$, these rates were from 0.74 to 0.94 . The average apparent alimentary conversion was from 10.1:1 on treatment $\mathrm{A}$ and $8.95: 1$ on treatment $\mathrm{B}$, during the 211 days of cultivation. The final weight in the non-live food treatment was $65 \%$ higher than in the live treatment and, apparently, Centropomus undecimalis accepted this kind of food well. However, it's necessary another studies to discover how to improve the management and obtain better growth rates for this species.

Key words: snoook, centropomidae, feed, culture and growth

\section{INTRODUÇÃO}

Centropomus undecimalis (Bloch, 1972), família Centropomidae, denominado vulgarmente camorim cabo-de-machado ou camorim açu, tem ampla distribuição na costa Atlântica Ocidental, sendo uma das espécies mais conhecidas e apreciadas na pesca costeira, desportiva e de subsistência (Silva, 1992). Sua popularidade é devido a excelente qualidade e sabor de sua carne (Tucker et al., 1985; Cerqueira, 1989), bem como pelo combate e resistência que oferece ao anzol quando fisgado.

Para que se possa cultivar camorins - também conhecidos de Sergipe a Santa Catarina como robalos - em condições intensivas, é necessário conhecer os vários aspectos que envolvem sua alimentação em condições de cativeiro. Para isto, um dos pontos principais é a aceitação de alimento inerte já que, sendo peixes carnívoros, podem apresentar alguma dificuldade neste aspecto (Bórquez-Ramíres, 1991), embora Cerqueira (1991) tenha comparado a resposta de $C$. undecimalis e $C$. parallelus à utilização de dietas natural e formulada, obtendo mais sucesso com o emprego da segunda. No Estado de Pernambuco, em estudo pioneiro, Silva (1976), utilizando guarus (Poecilidae) triturados na dieta de camorins juvenis, demonstrou a capacidade deste peixe em aceitar alimento natural inerte.

Especificamente sobre o cultivo de camorins e/ou de outros peixes carnívoros que venham a ser desenvolvidos no Canal de Santa Cruz (PE), uma possibilidade concreta para a obtenção de alimento natural inerte, aparentemente interessante, é o aproveitamento de organismos pequenos ou de pouco interesse comercial que compõem a fauna acompanhante da pesca do camarão, realizada no local durante considerável período do ano.

Face ao exposto, realizou-se o presente trabalho, cujo objetivo é subsidiar informações sobre o cultivo de camorins, em função do empre go de alimento vivo e dieta inerte composta de diversos organismos aquáticos triturados, como também sobre o grau de aceitabilidade a ambas. 


\section{MATERIAL E MÉTODOS}

A Base de Piscicultura Estuarina na qual realizou-se o presente trabalho pertence ao Departamento de Oceanografia da Universidade Federal de Pernambuco, estando localizada na Ilha de Itamaracá (PE), coordenadas de $7^{\circ} 48^{\prime} 15^{\prime \prime} \mathrm{S}$ e $34^{\circ} 51^{\prime} 30^{\prime \prime} \mathrm{W}$, litoral norte do Estado de Pernambuco, distante $50 \mathrm{~km}$ do Recife e separada do continente pelo Canal de Santa Cruz. No local, são realizados estudos sobre oceanografia e aquicultura, desde o início da década de setenta.

Para o experimento foram instalados, em um viveiro de $450 \mathrm{~m}^{2}$, quatro compartimentos iguais com $60 \mathrm{~m}^{2}$, divididos por cercas teladas (malha de $5 \mathrm{~mm}$ ) com 15 metros de comprimento e $1,5 \mathrm{~m}$ de altura. As estruturas foram presas a barrotes de madeiras e enterradas no solo a uma profundidade aproximada de $15 \mathrm{~cm}$. Os compartimentos foram instalados transversalmente no viveiro, isolando-se as extremidades. O mesmo é dotado de uma comporta de cimento com tábuas móveis, que funciona como meio de abastecimento e drenagem da água.

Para o estudo, foram delineados dois tratamentos, com duas repetições, distribuídos da seguinte maneira: a) para os testes onde administrou-se alimento vivo os compartimentos foram denominados $A_{I}$ e $A_{I I}$ e, b) para os testes com emprego de alimento inerte, os compartimentos foram denominados $\mathrm{B}_{\mathrm{I}}$ e $\mathrm{B}_{\mathrm{II}}$.

Os exemplares de camorins juvenis foram capturados com uma rede de arrasto (tipo mangote) com $15 \mathrm{~m}$ de comprimento x $1,5 \mathrm{~m}$ de altura, em um viveiro da própria base, por ocasião de sua despesca. Após a biometria (medições dos pesos e comprimentos totais), os peixes foram selecionados na faixa de comprimento de 12,0 a $19,0 \mathrm{~cm}$ e estocados com peso médio de $30 \mathrm{~g}$. Cada compartimento recebeu 48 peixes totalizando, no início do experimento, 192 indivíduos, numa densidade de 0,8 peixe $/ \mathrm{m}^{2}$.

A cada quatro dias a temperatura da água $\left({ }^{\circ} \mathrm{C}\right)$ era observada através de um termômetro de máxima e mínima, imerso no viveiro a uma profundidade de aproximadamente $20 \mathrm{~cm}$ da superfície. A salinidade (\%o) também foi aferida antes, e em seguida às renovações, (em intervalos aproximados de quinze dias) sendo utilizado para isto um salinômetro de bancada digital.

Mensalmente, de cada compartimento, eram capturados, ao acaso, no mínimo 25 $\%$ dos camorins, mediante rede de arrasto de 3,0 m de comprimento x 1,5 m de altura. Em seguida, os peixes eram transportados em baldes plásticos para o laboratório da Base, onde eram realizadas as medições de comprimento total (medida da extremidade da mandíbula inferior até a extremidade da nadadeira caudal), através de ictiômetro de acrílico com precisão de $1 \mathrm{~mm}$, e peso total individual, com uma balança com precisão de $1 \mathrm{~g}$.

Os organismos empregados no tratamento com presas vivas eram capturados periodicamente nos canais internos da Base de Piscicultura, com auxílio de uma rede de arrasto, posteriormente pesados e fornecidos aos peixes até a saciação, duas vezes ao 
dia, exceto aos domingos. Esta dieta constou principalmente de guaru (Poecilia vivipara Bloch e Schneider, 1801), camarão (Palaemonidae) e saúnas (Mugil sp.).

Nos ensaios com a utilização de alimento inerte foram ofertados organismos provenientes da fauna acompanhante da pesca do camarão realizada no Canal de Santa Cruz. Esses organismos eram triturados com uma máquina de moer caseira, pesados, acondicionados em sacos plásticos, congelados e fornecidos aos camorins "ad libitum" duas vezes ao dia, pela manhã e a tarde. A Tabela 1, apresenta a lista de organismos utilizados na preparação do alimento natural inerte.

Os dados coletados possibilitaram a estimação da taxa de crescimento específico (specific growth rate-SGR), Weatherley (1982),

$$
\left.\mathrm{SGR}=\left[\ln \left(\mathrm{W}_{\mathrm{t}}+\Delta_{\mathrm{t}}\right)-\ln \mathrm{W}_{\mathrm{t}}\right)\right] 100 / \Delta_{\mathrm{t}}
$$

onde:

$\mathrm{W}_{\mathrm{t}}=$ peso total médio dos exemplares no instante $(\mathrm{t})$ de cultivo;

$\mathrm{t}=$ tempo de cultivo; $\Delta_{\mathrm{t}}=$ intervalo de tempo entre as mensurações.

A biomassa final por tratamento foi determinada pelo somatório de todos os indivíduos ao final do experimento.

De posse da quantidade de alimento fornecido e da biomassa ganha durante o cultivo, foi estimada a taxa de conversão alimentar aparente (TCA), dada por:

TCA = quantidade de alimento fornecido $(\mathrm{g}) /$ ganho de peso líquido $(\mathrm{g})$

Tabela 1.: Organismos utilizados na preparação da dieta inerte, para C. undecimalis

\begin{tabular}{l|l}
\hline \multicolumn{1}{c|}{ Táxon } & \multicolumn{1}{c}{ Nome Vulgar } \\
\hline Eugerres $\mathrm{sp}$. & Carapeba \\
Engraulidae & Manjuba \\
Palaemonidae & Camarão \\
Gobiidae & Mingula \\
Eucinostomus $\mathrm{sp}$. & Carapicu \\
Mugil sp. & Saúna \\
Centropomus $\mathrm{sp}$ & Camorim \\
Poecilia vivipara & Guaru \\
Elopus saurus & Ubarana \\
Cactylopterus $\mathrm{sp}$. & Solhas \\
Callinectes $\mathrm{sp}$. & Siri \\
\hline
\end{tabular}

\section{RESULTADOS}

De acordo com a Tabela 2 observa-se que a temperatura da água apresentou valores oscilando entre $28,0^{\circ} \mathrm{C}$ e $35^{\circ} \mathrm{C}$, portanto, uma variação térmica de $7,0^{\circ} \mathrm{C}$.

A salinidade da água teve valores razoavelmente altos, uma vez que a maior parte do experimento ocorreu no período de estiagem, onde o índice pluviométrico é mais baixo. De acordo com a Tabela 3 , a salinidade no compartimento $\mathrm{A}_{\mathrm{I}}$, antes e após a renovação, variou de 17,2\%o em abril/96 a 39\%o em janeiro/96, sendo este o valor máximo encontrado, enquanto no $\mathrm{B}_{\mathrm{II}}$ os valores oscilaram de $15,6 \%$ em abril/96 a 36,5\% em 
janeiro/96, tendo ocorrido o menor índice nesse compartimento após a renovação da água.

Os resultados gerais obtidos sobre a criação de camorins em viveiro estuarino estão na Tabela 4.

Tabela 2 - Valores mínimos e máximos mensais de temperatura $\left({ }^{\circ} \mathrm{C}\right)$ da água no viveiro durante o período de cultivo.

\begin{tabular}{|c|c|c|c|}
\hline \multirow[t]{2}{*}{ Mês/Ano } & \multicolumn{3}{|c|}{ Temperatura ${ }^{\circ} \mathrm{C}$} \\
\hline & Máxima & - & Mínima \\
\hline Out/95 & 32,0 & - & 28,0 \\
\hline Nov/95 & 32,4 & - & 28,1 \\
\hline Dez/95 & 33,2 & - & 28,5 \\
\hline Jan/96 & 34,0 & - & 28,0 \\
\hline Fev/96 & 34,0 & - & 28,0 \\
\hline Mar/96 & 35,0 & - & 30,0 \\
\hline $\mathrm{Abr} / 96$ & 35,0 & - & 29,1 \\
\hline
\end{tabular}

Tabela 3 - Valores da salinidade da água nos compartimentos $\mathrm{A}_{\mathrm{Ie}} \mathrm{B}_{\mathrm{II}}$, antes e após, a renovação da água no viveiro.

\begin{tabular}{lcccc|}
\hline \multicolumn{1}{c}{ Período } & \multicolumn{4}{c|}{ Salinidade } \\
& Antes & Após & Antes & Após \\
\hline Outubro/95 & 34,0 & 32,0 & 33,4 & 31,3 \\
Novembro/95 & 32,8 & 31,2 & 32,4 & 31,0 \\
Dezembro/95 & 34,5 & 32,7 & 34,1 & 32,1 \\
Janeiro/96 & 39,0 & 33,7 & 36,5 & 33,1 \\
Fevereiro/96 & 35,9 & 31,8 & 35,0 & 31,2 \\
Março/96 & 32,6 & 31,1 & 32,6 & 30,7 \\
Abril/96 & 19,5 & 17,2 & 17,9 & 15,6 \\
\hline
\end{tabular}

Tabela 4 - Dados gerais sobre a criação experimental do camorim, Centropomus undecimalis (Bloch, 1792).

\begin{tabular}{l|c|c|c|c}
\hline \multicolumn{1}{c|}{ Dados } & \multicolumn{4}{c}{ Compartimentos } \\
\hline & AI & BI & AII & BII \\
\hline Taxa de estocagem (ind./m ${ }^{2}$ ) & 0,8 & 8,8 & 8,8 & 0,8 \\
Dias de cultivo & 211 & 211 & 211 & 211 \\
Peso médio inicial (g) & 29,87 & 29,63 & 30,91 & 31,89 \\
Peso médio final (g) & 117,2 & 217,7 & 105,7 & 151,6 \\
Comprimento médio inicial (cm) & 14,8 & 14,7 & 14,9 & 15,1 \\
Comprimento médio final (cm) & 24,7 & 28,9 & 23,5 & 26 \\
Biomassa inicial (g) & 1433,6 & 1422,3 & 1483,7 & 1550,9
\end{tabular}

Trab. Oceanog. Univ. Fed. PE, Recife, 27 (1): 115-127, 1999. 


\begin{tabular}{l|c|c|c|c} 
Biomassa final (g) & 4453,6 & 8708,0 & $3,699,5$ & 7276,8 \\
Biomassa ganha (g) & 3019,6 & 7285,6 & 2215,6 & 5725,7 \\
Sobrevivência (\%) & 79 & 83 & 73 & 100 \\
Peso ganho / indivíduo (g/dia) & 0,37 & 0,86 & 0,3 & 0,56 \\
Taxa de conversão alim. aparente & $8,3: 1$ & $7,7: 1$ & $11,9: 1$ & $10,2: 1$ \\
Taxa de crescimento específico (SGR) & $8,3: 1$ & $7,7: 1$ & $11,9: 1$ & $10,2: 1$ \\
\hline
\end{tabular}

As taxas de sobrevivência foram satisfatórias, com os maiores percentuais ocorrendo nos compartimentos $\mathrm{B}_{\mathrm{I}}$ e $\mathrm{B}_{\mathrm{II}}$ com 83 e $100 \%$, respectivamente, e o menor verificado no $\mathrm{A}_{\mathrm{II}}$ com $73 \%$.

Os valores de conversão alimentar aparente apresentaram-se relativamente altos, sendo o maior valor 11,9:1 no compartimento $\mathrm{A}_{\mathrm{II}}$ e o menor 7,7:1 no $\mathrm{B}_{\mathrm{I}}$.

As taxas de SGR foram 0,64 e 0,58 nos compartimentos $A_{I}$ e $A_{I I}$, respectivamente, observando-se que nos três primeiros meses os resultados foram melhores, ocorrendo uma queda bastante acentuada no final do cultivo. Nos compartimentos $B_{I}$ e $B_{I I}$ os resultados foram similares, com o valor final de SGR superior no $B_{\text {II }}$, equivalendo a 0,94 .

De acordo com a Figura 1, observa-se que houve uma maior ocorrência dos camorins alimentados com dietas inertes nas classes de peso maiores, comparativamente com os peixes alimentados com dieta viva, nas quais as maiores freqüências se concentram nas classes de pesos menores, confirmando a boa adaptabilidade dessa espécie ao alimento inerte. 


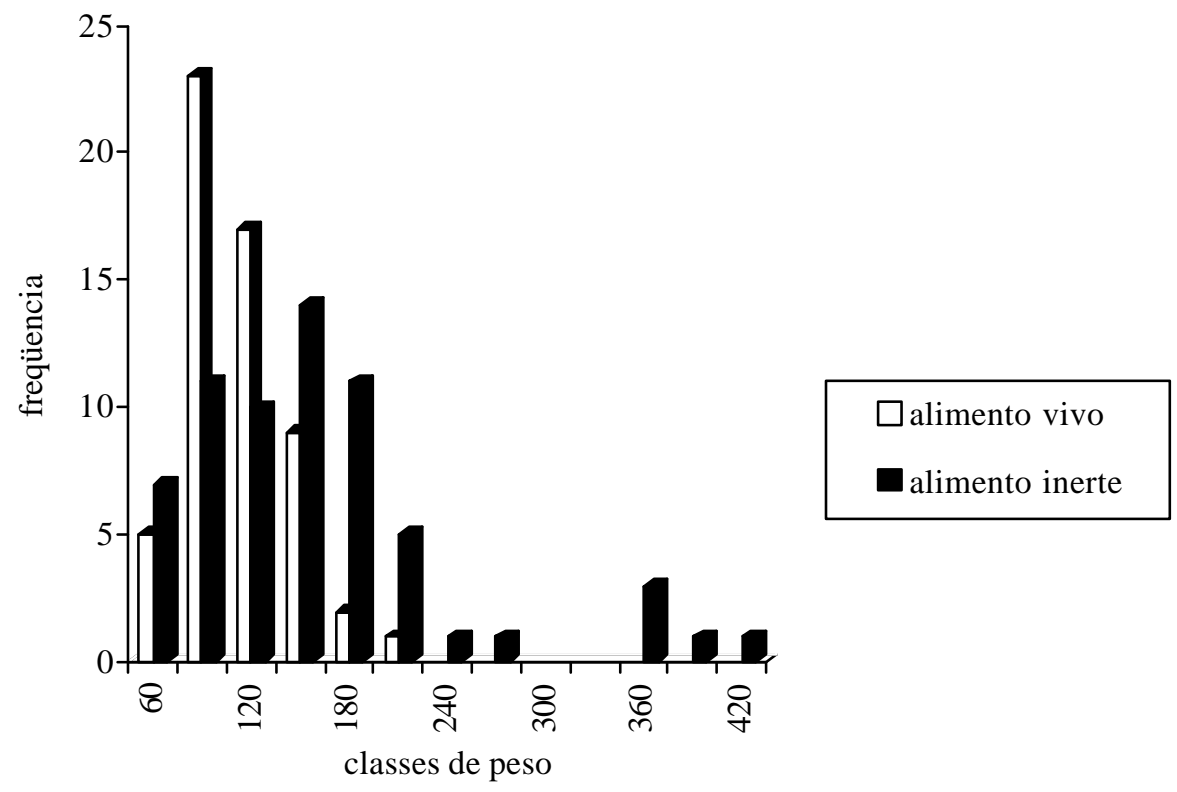

Figura 1. - Distribuição de frequiência final dos camorins por classe de peso, nos dois tratamentos estudados.

\section{DISCUSSÃO}

Desde que há influência das variáveis ambientais sobre o desenvolvimento das espécies cultiváveis e de que os efeitos dessas, quando detectados a tempo, podem ser minimizados, os estudos hidrológicos têm fundamental importância, principalmente quanto à temperatura, salinidade, oxigênio dissolvido, transparência e amônia.

A temperatura, apesar de ser um fator que influencia decisivamente os processos fisiológicos dos organismos aquáticos, não chega a afetar diretamente o desenvolvimento das espécies cultivadas em ambientes estuarinos da Região Nordeste (Rocha et al., 1981). Cerqueira (1991) observou que camorins nas temperatura em torno de $29^{\circ} \mathrm{C}$ ou acima, alimentam-se bem, enquanto temperaturas abaixo de $18{ }^{\circ} \mathrm{C}$ inibem a sua busca por alimento. Nas nossas condições, Santos (1994) obteve variação térmica de $4,4^{\circ} \mathrm{C}$, com máxima de $31,3^{\circ} \mathrm{C}$ e mínima de $26,9^{\circ} \mathrm{C}$, trabalhando em viveiro contíguo ao do presente estudo. Neste cultivo a temperatura variou de 28,0 a $35,0^{\circ} \mathrm{C}$ com amplitude térmica de $7,0^{\circ} \mathrm{C}$, inferior a registrada por Silva (1976) que obteve variação térmica de 8,5 ${ }^{\circ} \mathrm{C}$, estudando a mesma área, sem haver, aparentemente, problemas para os peixes. No

Trab. Oceanog. Univ. Fed. PE, Recife, 27 (1): 115-127, 1999. 
entanto, comparado-se com outros dados disponíveis, tais valores podem ser considerados elevados, uma vez que Macêdo et al. (1980) obtiveram índice de variação térmica de apenas $3,5^{\circ} \mathrm{C}$, com máxima de $31,09^{\circ} \mathrm{C}$ e mínima de $26,6{ }^{\circ} \mathrm{C}$ em viveiros da mesma Base. Millán-Q (1989) cultivando camorins em tanques na Venezuela obteve amplitude térmica em torno de $2,5^{\circ} \mathrm{C}$, o mesmo tendo sido verificado por Rocha e Okada (1980), Okada et al. (1980) e Maia et al. (1980), que constataram variações térmicas em média de $4,5^{\circ} \mathrm{C}$ em cultivos de camorins nos viveiros da Base de Pis cicultura Estuarina de Itamaracá.

Como a renovação da água no presente trabalho ocorreu, na maioria das vezes, em intervalos de 15 dias, pelo fato das preamares não alcançarem os níveis desejáveis fora desses intervalos, pode ter ocorrido aumento na temperatura da água conjugado com hipersalinidade no viveiro, uma vez que a grande quantidade de radiação solar incidida sobre este biótopo, aumenta consideravelmente o processo de evaporação e, conseqüentemente, a concentração de sal. Por seu lado, a tolerância do camorim $C$. undecimalis a ambientes salinos é vasta, pois a espécie pode ser encontrada desde ambientes com água doce à salinidades do mar. Silva (1976), durante o período de março de 1970 a março de 1975, observou variações de 9,54 \%o a 36,54 \%o em viveiros da Base de Itamaracá, onde é bem nítida a diferença de salinidade entre os períodos considerados chuvosos e secos. Rocha e Okada (1980), Maia et al. (1980) e Okada et al. (1980) constataram em seus trabalhos que a salinidade máxima foi sempre acima de $32 \%$ o em períodos secos, e as mínimas variando de 14,0 a 19,4\%o, em períodos chuvosos, aproximando-se dos resultados deste estudo. Apesar do cultivo ter sido realizado durante apenas um mês considerado chuvoso, observa-se que as salinidades mais altas ocorreram nos meses de verão ou seja, outubro de 1995 a março de 1996, com valor máximo de $39 \%$ em janeiro de 1996 e valores bem inferiores no mês de abril do mesmo ano. Todavia, Millán-Q (1989), cultivou camorins em salinidades mais altas, com máxima de $41,2 \%$ e mínima de $38,5 \%$, obtendo $100 \%$ de sobrevivência e bom desenvolvimento. A Tabela 5 mostra os resultados obtidos por alguns autores em cultivo de camorins, em relação a temperatura e salinidade da água.

Fica evidenciado que, tanto os resultados obtidos neste estudo quanto os observados por outros autores, corroboram os verificados por Macêdo et al. (1980), segundo os quais as variações de temperatura e salinidade estão associadas às estações do ano onde a insolação e os índices pluviométricos são extremos. Assim, confirma-se a capacidade do camorim em tolerar condições adversas, sem que ocorra danos aparentes ao seu desenvolvimento.

A propósito do oxigênio dissolvido, Macêdo et al. (1980), estudando viveiros da Base de Itamaracá, relatam concentrações de 2,5 ml/1 a 5,49 ml/1. Santos (1994), trabalhando em viveiro contíguo ao do presente estudo, determinou teores mínimos de 4,9 ml/1 em período semelhante. Segundo Rocha e Okada (1980), os centropomídeos são peixes eurihalinos e euritérmicos, porém, mesmo tolerando níveis de oxigênio inferiores a $1 \mathrm{ml} / \mathrm{l}$, eles requerem suprimentos de 4,0 a $5,0 \mathrm{ml} / \mathrm{l}$ de oxigênio para se desenvolverem normalmente. Acredita-se, portanto, que o índice de oxigênio dissolvido nesse viveiro, 
por analogia aos autores mencionados, esteve dentro dos padrões normais para o crescimento do camorim.

Tabela 5. - Comparação dos resultados obtidos por alguns autores no cultivo de camorins, com relação a temperatura e salinidade.

\begin{tabular}{|c|c|c|c|c|}
\hline \multirow[t]{2}{*}{ Autor } & \multicolumn{2}{|c|}{ Temperatura $\left({ }^{\circ} \mathrm{C}\right)$} & \multicolumn{2}{|c|}{ Salinidade $(\% o)$} \\
\hline & máx & $\min$ & máx & $\min$ \\
\hline Okada et al. (1980) & 30.5 & 25.5 & 33.5 & 14.0 \\
\hline Rocha e Okada (1980) & 31.8 & 26.9 & 32.6 & 19.4 \\
\hline Maia et al. (1980) & 30.6 & 25.7 & 31.0 & 19.0 \\
\hline Millán-Q (1989) & 29.0 & 26.5 & 42.2 & 38.5 \\
\hline Santos (1994) & 31.3 & 26.9 & 40.37 & 23.57 \\
\hline Presente trabalho & 35.0 & 28.0 & 39.0 & 15.6 \\
\hline
\end{tabular}

Em aqüicultura é importante a determinação do crescimento dos indivíduos, em função da densidade populacional e conseqüentes efeitos da competição por alimento, pois o crescimento em comprimento e peso depende do suprimento alimentar, o qual está inteiramente relacionado à densidade (Santos, 1994).

Um dos pontos mais importantes na aqüicultura é o emprego de rações objetivando melhorar o aproveitamento das áreas cultivadas, uma vez que estas aumentam a disponibilidade de alimentos para os peixes, proporcionando-lhes desenvolvimento precoce e atuando positivamente sobre a sobrevivência desses, considerando-se que este tipo de alimentação deve funcionar, porém, como um suplemento do alimento natural (Rocha et al., 1981).

De acordo com Benetti (1983), na aqüicultura, o desperdício de energia empregado pelo animal na captura do alimento deve ser mínimo, direcionando-se o gasto de energia para o melhor desenvolvimento do organismo cultivado.

No presente estudo, os resultados alcançados com relação ao crescimento específico (SGR) utilizando alimento vivo foram de 0,58 a 0,64 , com densidade de 0,8 ind $/ \mathrm{m}^{2}$, portanto inferiores aos atingidos por Millán-Q (1989) que, ao cultivar $C$. undecimalis em tanque de parede de cimento e fundo de terra, obteve valor de 1,15 com densidade de $0,62 \mathrm{ind} / \mathrm{m}^{2}$ em período de 180 dias. Por outro lado, valores satisfatórios também foram encontrados por Silva (1992) ao cultivar camorins associados à tilápias, na função de controladores de desovas indesejáveis, obtendo valores para SGR de 1,03 a $1,93 \mathrm{com}$ uma densidade de $0,30 \mathrm{ind} / \mathrm{m}^{2}$, em viveiro de água doce. Bom crescimento também foi constatado em um monocultivo realizado também em água doce, em 210 dias, com SGR de 1,24 para densidade de $0,33 / \mathrm{m}^{2}$ e dieta viva diversificada (Silva, Rosa e Carmo, 1992).

A respeito da taxa de sobrevivência dos camorins cultivados, Millán-Q (1989), alcançou valor de 100\%, com um ganho de peso de 3,28 g/dia (indivíduo). Este valor foi 
bastante superior aos encontrados por outros autores que cultivaram estes peixes com alimentos vivos. No presente estudo, o tratamento no qual foi utilizado alimento vivo na dieta dos peixes, resultou numa sobrevivência média de $76 \%$ e o ganho de peso de 0,33 g/dia (indivíduo), valores comparáveis aos encontrados por Okada et al. (1980), Maia et al. (1980) e inferior aos de Rocha e Okada (1980), Santos (1994), Silva (1992) e Silva et al. (1992), que obtiveram valores médios de $100 \%$ de sobrevivência e ganho de peso de $1,17 \mathrm{~g} / \mathrm{dia}$, ressalvando-se as diferentes condições de cultivo, ocorrida nos respectivos experimentos.

Tendo em vista que os resultados citados, comparativamente aos do presente trabalho, ocorreram em períodos maiores e densidades menores, é razoável que os valores das taxas de crescimento sejam um pouco superiores, uma vez que a competição pelo alimento torna-se menor. Todavia, observando-se os trabalhos de Millán-Q (1989) e Silva et al. (1992), nos quais tanto os períodos de cultivo como as densidades são próximas, novamente observa-se que as taxas de crescimento do presente trabalho são menores, o que pode ser atribuído, provavelmente, à deficiência no manejo alimentar e, principalmente à condição geral do viveiro, o qual possuía uma vasta camada de lama no fundo.

No que concerne a utilização de dietas inertes, Silva (1976), em trabalho pioneiro no país, ao cultivar camorins em tanques-rede alimentados com guarus triturados, obteve taxas de crescimento específico desde 0,57 a $1,03 \%$, não ocorrendo muita variação em relação aos resultados deste trabalho, onde as SGR oscilaram de 0,94 a 0,78 nos compartimentos $\mathrm{B}_{\mathrm{I}}$ e $\mathrm{B}_{\mathrm{II}}$. Cerqueira (1991), observando o crescimento de camorins $C$. undecimalis e $C$. parallelus, obteve valores de SGR variando de 0,2 a 0,8 com dietas formuladas. Tuckey (1987), testando dietas formuladas, obteve taxas variando de 0,54 a 2,08 em cultivo de camorins C. undecimalis em água doce. Franciski et al. (1996), observando o desenvolvimento de $C$. parallelus com 64 dias de idade obtiveram SGR de 1,36 e de 1,6, enquanto os camorins com idade de 369 dias de idade, apresentaram valores de SGR igual a 0,31. Por seu lado Borba et al. (1996) ao comparar o desempenho de diferentes dietas num "desmame" do C. parallelus, não observaram grandes diferenças no desenvolvimento dos peixes, apenas ressaltando a maior dificuldade na aceitação da ração composta só de farinhas.

Cerqueira e Macchiavello (1994), comparando o crescimento de C. undecimalis juvenis, obtiveram taxa de crescimento de 0,79 com ganho de peso de $1,81 \mathrm{~g} / \mathrm{dia}$ valores referentes à dieta experimental para camorins, a qual apresentou valores superiores à dieta comercial para truta. Borba et al. (1994), utilizando fontes protéicas diferentes nas dietas artificiais para $C$. palallelus, obtiveram taxa de crescimento de 1,74 com camorins estocados com idade de 87 dias e SGR de 1,80 com idade de 145 dias, com dieta a base de farinha de peixe, sendo esses valores superiores aos resultados encontrados pelo mesmo autor com peixes da mesma idade e as mesmas condições, utilizando uma dieta a base de proteína animal.

Os valores da taxa de conversão alimentar obtidos no presente trabalho, relativamente altos quando comparados com outros cultivos e espécies, pode ser atribuído a perdas no fornecimento "ad libitum", principalmente com a dieta inerte na 124 
fase inicial do cultivo. Comparativamente a Santos (1994), que forneceu guarus a $C$. undecimalis em gaiolas, os resultados são equivalentes.

A taxa de sobrevivência dos camorins cultivados por Franciski et al. (1996) foi de 50,28\%, enquanto Silva (1976) obteve valor médio nos três testes de $91,9 \%$ com peixes cultivados em tanques-rede, com a utilização de guaru triturado como alimento. Neste experimento, no tratamento onde administrou-se dieta inerte, a taxa de sobrevivência média foi de 91,5\%, valor satisfatório se comparado com outros autores. Cerqueira (1991) obteve valor médio, em cinco testes, de $85 \%$ de sobrevivência e posteriormente o mesmo autor, três anos após, alcançou uma taxa de sobrevivência de 100\% em 132 dias de cultivo, com uma dieta comercial a base de proteína animal, para trutas. Foi verificado também que o ganho de peso médio de 0,71g/dia, neste experimento, mostrou-se superior aos valores médios encontrados por Cerqueira (1991), em cinco testes e por Franciski et al. (1996) em dois testes, com valor médio de 0,23 e 0,17, respectivamente, enquanto comparando-se com Cerqueira e Macchiavello (1994) e Silva (1976), os quais obtiveram ganho de peso de 1,81 e 1,48 g/dia (média de dois e três testes, respectivamente), verifica-se que os valores encontrados foram bastante inferiores.

A análise dos dados obtidos evidencia um melhor rendimento nos tratamento onde administrou-se dieta inerte em relação ao cultivo com alimento vivo, mesmo comparativamente a outros dados de manejos. Percebe-se ainda que o crescimento do camorim com dietas inertes pode tornar-se uma prática bem sucedida, corroborando os resultados obtidos por Bórquez-Ramíres (1991), ao concluir sobre a viabilidade de fornecer dietas inertes para $C$. undecimalis a partir da introdução de substâncias químicas como atrativos. Com efeito, a média dos pesos finais da dieta a base de organismos estuarinos triturados foi superior à dieta viva na ordem de $65 \%$. No entanto, ainda são necessários maiores estudos para que se possa encontrar uma dieta que supra todas as necessidades nutricionais do peixe.

\section{CONCLUSÃO}

Como decorrência do presente estudo, basicamente se conclui que, há necessidade de se aprofundar os conhecimentos sobre Centropomus undecimalis no que concerne a sistemas de cultivos e manejo alimentar, sendo ainda possível constatar que: 1) camorins, em confinamento, aceitam bem alimento inerte;

2) o tratamento com dieta inerte (a base de organismos estuarinos triturados) proporcionou um ganho de peso $65 \%$ superior ao da dieta viva;

3) a decisão sobre a escolha de uma das dietas testadas carece, também, de análise econômica. 


\section{REFERÊNCIAS BIBLIOGRÁFICAS}

BENETTI, D. A aquacultura como alternativa e complemento da pesca Boletim FCBN, Rio de Janeiro, v.18, p.85-95, 1993

BORBA, M. R.; CERQUEIRA, V. R.; NOLLI, N. L. et al. Utilização de diferentes fontes proteícas na dieta artificial de juvenis de robalo (Centropomus parallelus) em cultivo experimental. In: SIMPÓSIO BRASILEIRO DE AQÜICULTIRA, 8., ENCONTRO BRASILEIRO DE PATOLOGIA DE ORGANISMOS AQUÁTICOS, 3., 1994, Piracicaba. Resumos... Piracicaba: José Eurico Possebon Cyrino, José Carlos de Moura, 1994. p. 43.

.; SEIFFERT, E. G.; CERQUEIRA, V. R. Comparação do desempenho de diferentes dietas no desmame do robalo Centropomus parallelus. In: REUNIÃO ESPECIAL DA SBPC, 3., 1996, Florianópolis. Resumos... Florianópolis: :SBPC, 1996. p. 194.

BÓRQUEZ-RAMÍRES, A. S. Comportamento alimentar do juvenil do robalo, Centropomus undecimalis, Bloch, 1792 (Pisces:Centropomidae), face a atrativos químicos e extratos aquosos animais. Florianópolis, 1991. 99 p. Dissertação (Mestrado em Aqüicultura) - Universidade Federal de Santa Catarina, 1991.

CERQUEIRA, V. R. Análise e perspectiva para o cultivo do robalo, Centropomus sp., no litoral brasileiro. Florianópolis, 1989. 15 p. Dissertação (Monografia de Prof. Assistente em Piscicultura) - Universidade Federal de Santa Catarina, 1989.

- Observações preliminares sobre o crescimento de juvenis de robalo, Centropomus undecimalis, com dietas naturais e artificiais. 1991. In: CONGRESSO BRASILEIRO DE ENGENHARIA DE PESCA, 7., p. 85-94, 1991, Santos. Anais... São Paulo, 1991.

.; MACCHIAVELlO, J. A. Comparação do crescimento de juvenis do robalo (Centropomus undecimalis) alimentados com uma dieta comercial para truta. In: SIMPÓSIO BRASILEIRO IE AQÜICULTURA, 8., ENCONTRO BRASILEIRO DE PATOLOGIA DE ORGANISMOS AQUÁTICOS, 3., 1994, Piracicaba. Resumos... Piracicaba: José Eurico Possebon Cyrino, José Carlos de Moura, 1994. p. 43.

FRANCISKI, N. ; MOMM, E. R.; CERQUEIRA, V. R. Engorda experimental de robalo, Centropomus parallelus, com dietas artificiais. In: REUNIÃO ESPECIAL DA SBPC, 3., 1996, Florianopólis. Resumos... Florianópolis: :SBPC, 1996. p. 194.

MACÊDO, S. J. de; CAVALCANTI, L. B.; COSTA, K. M. P. Variação dos parâmetros físico-químicos em viveiros de cultivo da Ilha de Itamaracá (Pernambuco-Brasil). In: SIMPÓSIO BRASILEIRO DE AQÜICULTURA, 1., 1978, Recife. Anais... Rio de Janeiro: Academia Brasileira de Ciências, 1980. p. 73-85.

MAIA, E. de P.; ROCHA, I. de P.; OKADA, Y. Cultivo arraçoado de curimã (Mugil brasiliensis Agassiz, 1929) em associação com tainha (Mugil curema Valenciennes, 1836) e camorim (Centropomus undecimalis Bloch, 1972) em viveiros estuarinos de Itamaracá-PE. In: SIMPÓSIO BRASILEIRO DE AQÜICULTURA, 1., 1978, Recife.

Anais... Rio de Janeiro: Acadêmia Brasileira de Ciências, 1980. p.141-149.

MILLÁN-Q, J. R. Resultados del crescimiento del robalo Centropomus undecimalis Bloch, 1792 (Pisces: Centropomidae) en estanques. Rev. Lat. Acui., Lima, n. 41, p. 4556, sept, 1989. 
OKADA, Y.; MAIA, E. de P.; ROCHA, I. de P. Cultivo arraçoado de tainha (Mugil curema Valenciennes, 1836) em associação com robalo (Centropomus undecimalis Bloch, 1792) e carapeba (Eugerres brasilianus Curvier, 1830) em viveiros estuarinos de Itamaracá-PE. In: SIMPÓSIO BRASILEIRO DE AQÜICULTURA, 1., 1978, Recife. Anais ... Rio de Janeiro: Acadêmia Brasileira de Ciências, 1980. p. 131-139.

ROCHA, I. de P.; OKADA, Y. Experimentos de policultivo em curimã (Mugil brasiliensis Agassiz, 1829) e camorim Centropomus undecimalis Bloch, 1792) em viveiros estuarinos (Itamaracá-Pernambuco). In: SIMPÓSIO BRASILEIRO DE AQÜICUlTURA, 1., 1978, Recife. Anais... Rio de Janeiro: Academia Brasileira de Ciências, 1980. p. 163-173.

.; MAIA, E. de P.; PARANAGUÁ, M. N. et al. Pis cicultura estuarina: aspectos técnicos do cultivo. In: CONGRESSO BRASILEIRO DE ENGENHARIA DE PESCA, 2., 1981, Recife. Anais... Recife: Associação dos Engenheiros de Pesca de Pernambuco, 1981. p. 85-100.

SANTOS, G. A. C. Crescimento de camurins jovens, Centropomus undecimalis (Bloch, 1792) em viveiros-rede fixos. Recife, 1994, 133p. Dissertação (Mestrado em Oceanografia Biológica) - Universidade Federal de Pernambuco, 1994.

SILVA, A. L. N. da. Efeito da predação do Centropomus undecimalis (Bloch, 1792) PISCES: Centropomidae - sobre a tilápia Oreochromis niloticus (Linnaeus, 1758) cultivados em viveiros de água doce. Florianópolis, 1992. 105p. Dissertação (Mestrado em Aqüicultura) - Universidade Federal de Santa Catarina, 1992.

SILVA, A. L. N.; ROSA, M. C. G.; CARMO, J .L. Crescimento do robalo Centropomus undecimalis (Bloch, 1792) (Pisces, Centropomidae) em condições de água doce. In: SIMPÓSIO BRASILEIRO DE AQÜICULTURA, 7., 1992, Peruíbe. 1992.

SILVA, J. E. da. Fisioecologia do camorim, Centropomus undecimalis (Bloch, 1792). Estudo experimental em ambiente confinado. São Paulo, 1976. 101p. Tese (Doutorado em Ciências) - Universidade de São Paulo, 1976.

TUCKEY JR, J. W.; LANDAU, M.; FAULKNER, B. E. Culinary value and composition of wild and captive common snook Centropomus undecimalis. Florida Scientist, Fort Pierce, v. 48, n. 4, p. 193-196, 1985.

TUCKEY JR, J. W. Snook and tarpon snook culture and preliminary evalution for commercial farming. Progressive Fish-Culturist, Bethesda, n. 49, p. 49-57, 1987.

WEATHERLEY, A. H. Growth and ecology of fish populations. London: Academic Press, 1982. 193p. 